\title{
Conductas \\ de autorregulación infantil ante dos tareas
}

\section{Cecilia Méndez Sánchez ${ }^{1}$, Pedro Palacios Salas ${ }^{1}$, Miguel Ángel Sahagún Padilla ${ }^{1}$ y Alicia Edith Hermosillo de la Torre ${ }^{1}$}

\section{Introducción}

Las reacciones que tienen los niños frente a una tarea pueden ser explicadas desde la forma en que la perciben. Esta percepción va a tener sus efectos en su motivación, determinando sus expectativas, reacciones emocionales y permanencia en la tarea, entre otras cosas (Weiner, 1986). Al evaluar la percepción que tienen los niños respecto a una tarea a la que se enfrentan, podemos encontrar que no sólo se considera la naturaleza de la tarea sino otras variables que se encuentran inmersas, como su nivel de dificultad y las demandas que se le hagan respecto a la tarea (Feigenbaum, 2004). Sus reacciones pueden verse desde aspectos verbales o bien desde aspectos no verbales. En los aspectos verbales encontramos elementos de análisis como el habla privada (Berk, 1986) y el habla social, con lo cual harán referentes a aspectos motivacionales, afrontamiento de la tarea, atribuciones de causalidad y regulación emocional; mientras que en los aspectos no verbales, nos pueden dar cuenta de las

1 Universidad Autónoma de Aguascalientes. 
estrategias de afrontamiento o comportamientos que realiza el niño para resolver la tarea y aspectos de regulación emocional.

Las tareas escolares, además de estar asociadas con aspectos de valoración académica, también lo están con aspectos de valoración personal, lo que lleva a las personas a realizar atribuciones de causalidad en función del resultado y a presentar una serie de comportamientos que se derivan de ellas. Se han observado diferencias comportamentales en relación con la naturaleza de las tareas, específicamente cuando éstas son de matemáticas o bien cuando la naturaleza es no académica (Boekaerts, Otten y Voeten, 2003), como por ejemplo, las lúdicas.

Según Covington (1984, 1992, 2000a), la autovaloración, entendida como una necesidad básica, lleva a las personas a salvaguardar su autoestima, misma que se ve vulnerada cuando se enfrenta a tareas en las que es posible que fracase, presentando con ello distintos comportamientos defensivos con el fin de mantener su sentimiento de valía personal, su autoimagen positiva y de pertenencia a una sociedad que valora la capacidad de los individuos (Covington, 2000b).

En los estudios en los que se han comparado tareas de naturaleza distinta (académica y lúdica), se ha encontrado que hay diferencias marcadas en el comportamiento verbal de los niños. De manera específica, cuando se analizó el Habla Privada (HP) de los niños, se encontró que ante una tarea lúdica, los niños se permitían hablar sobre aspectos irrelevantes a la tarea, mientras que cuando la tarea era de matemáticas, los niños tendían a presentar menos comportamientos irrelevantes y a presentar más verbalizaciones que les ayudaran a estructurar la tarea (Méndez-Sánchez, 2012). En este estudio se busca establecer las diferencias en el comportamiento no verbal que los niños presentan ante dos tareas cuya naturaleza es distinta.

\section{Método}

Participaron en este estudio 72 niños de educación primaria (2, 4 y 6 grados) con igual número de hombres y de mujeres en cada 
grupo, procedentes de tres centros educativos (dos escuelas públicas y una escuela privada) de la ciudad de Aguascalientes. La edad de los participantes osciló entre los 7 años 5 meses hasta los 13 años 7 meses, con una media de 10 años 2 meses.

Los participantes debían resolver dos tareas distintas (una tarea lúdica y una tarea académica) bajo dos situaciones de demanda: cooperación y competición. La tarea lúdica consistía en la resolución de rompecabezas del Tangram, donde en una lámina se presentaba el modelo que se tenía que reproducir, dividida en siete niveles de dificultad (De Dios, 2004). A cada niño se le dio solamente la lámina para reproducir y la cantidad exacta de piezas que necesitaba para realizar en función del nivel de dificultad en el que fue calibrado. La tarea académica consistió en la resolución de problemas matemáticos, los cuales fueron seleccionados de los libros de texto gratuito de la Secretaría de Educación Pública (SEP). La dificultad de la tarea académica también fue calibrada para cada niño.

Se videograbó a los niños mientras resolvían las tareas y posteriormente se codificaron las conductas que acompañaron la tarea. Se utilizó el sistema de codificación de "Conductas que acompañan la tarea", adaptado por Pechman (1978), donde se incluyen las conductas físicas que acompañan la tarea, ya sea para reducir la tensión (automanipulación, morder o masticar, movimientos rítmicos y movimientos del cuerpo) o bien para facilitar la tarea (tomar puntos de referencia, hacer gestos, mirar y mover objetos).

\section{Resultados}

Para asegurarnos de la correcta aplicación de los sistemas de codificación utilizados, se obtuvo la confiabilidad interjueces: 90.59\% en las conductas para reducir la tensión y $97.36 \%$ en las conductas para facilitar la tarea.

Ante la tarea de Tangram se presentaron 1,117 comportamientos que acompañan la tarea, de los cuales 763 son comportamientos para reducir la tensión (333 en la demanda de 
cooperación y 430 en la demanda de competición) y 354 para facilitar la tarea (154 en la demanda de cooperación y 200 en la demanda de competición).

Ante la tarea de matemáticas se presentaron 2,859 comportamientos que acompañan la tarea, de los cuales 1,321 son comportamientos para reducir la tensión (640 en la demanda de cooperación y 681 en la demanda de competición) y 1,538 para facilitar la tarea (753 en la demanda de cooperación y 785 en la demanda de competición).

Los comportamientos para reducir la tensión que se emitieron con más frecuencia en los distintos grados escolares, ante ambas tareas y en las dos demandas, fueron las conductas de automanipulación y los movimientos del cuerpo. Las conductas de automanipulación y de mirar tendieron a ser mayores ante la tarea de matemáticas que ante la tarea lúdica en ambas demandas. Sólo la conducta de morder tendió a ser más emitida ante la tarea en todos los grados escolares y ante las dos demandas.

Con relación a las conductas para facilitar la tarea, los gestos se presentaron principalmente en la tarea de matemáticas, independientemente de la demanda hecha para realizar la tarea y del grado escolar. Tomar puntos de referencia es un comportamiento que tiende a presentarse ante ambas tareas, pero en la tarea lúdica fue más utilizado ante la demanda de competencia y en la tarea académica lo fue ante la demanda de cooperación. Finalmente, la conducta de mirar se presentó en ambas tareas, siendo mayor su incidencia en la tarea académica, especialmente ante la demanda de cooperación.

\section{Conclusiones}

La tarea académica llevó a los niños a presentar más comportamientos tanto para reducir la tensión como para facilitar la tarea en comparación con la tarea lúdica. La combinación naturaleza de la tarea y demanda tienen un mayor efecto en la realización de estos comportamientos, es decir, que cuando se agrega una presión adicional a la tarea y esta presión involucra una evaluación hacia 
el niño. Tanto en la tarea académica como en la tarea lúdica los niños tienden a presentar más comportamientos para reducir la tensión. En este sentido, podemos ver cómo las situaciones competitivas y de evaluación que se producen en la escuela tienen implicaciones importantes en la autovaloración de la competencia académica de los alumnos y cómo los comportamientos que tienen al estar realizando una tarea les ayudan en el mantenimiento de la autovaloración tal como lo planteaba Covington (1992), además de que lleva a los niños a realizar más comportamientos para facilitar la tarea ante la tarea académica que ante la tarea lúdica (matemáticas $=1538$, Tangram $=354$ ), ello debido quizá a la exigencia de hablar cada vez menos mientras se está trabajando.

\section{Referencias}

Berk, L.E. (1986). Relationship of Elementary School Children's Private Speech to Behavioral Accompaniment to Task, Attention, and Task Performance. Developmental Psychology, 22(5), 671-680.

Boekaerts, M., Otten, R. y Voeten, R. (2003). Examination Performance: Are Student's Causal Attributions School-Subject Specific? Anxiety, Stress and Coping, 16(3), 331-342. Covington, M.V. (1984). The Motive for Self-Worth (77-113). En R.E. Ames y C. Ames, Research on Motivation in Education, Vol. 1. Nueva York: Academic Press.

Covington, M.V. (1992). Making the Grade: A Self-Worth Perspective on Motivation and School Reform. Nueva York: Cambrigde University Press.

Covington, M.V. (2000a). La voluntad de aprender: Guía para la motivación en el aula. Madrid: Alianza. Trabajo original publicado en 1998.

Covington, M.V. (2000b). Goal Theory, Motivation and School Achievement: An Integrative Review. Annual Review of Psychology, 51, 171-200.

De Dios, M.J. (2004). La función motivacional del habla privada: una perspectiva vygotskiana para el estudio del desarrollo 
de la motivación en educación. Tesis doctoral. Universidad Autónoma de Madrid.

Feigenbaum, P. (2004). Empirical Evidence of The Many Uses of Private and Inner Speech. En I. Montero (Ed.), Current Research Trends in Private Speech Proccedings from the First International Symposium on Self-Regulatory Functions of Language (67-90). Madrid: Publicaciones de la Universidad Autónoma de Madrid.

Méndez-Sánchez, C. (2012). Lenguaje y desarrollo: Estudio del desarrollo del pensamiento atribucional a través del habla privada. Tesis doctoral. Universidad Autónoma de Madrid. Pechman, E.M. (1978). Spontaneous Verbalization and Motor Accompaniment to Children's Task Orientation in Elementary Classrooms. Dissertation Abstracts International, Vol. 39 (2-A), 786.

Weiner, B. (1986). An Attributional Theory of Motivation and Emotion. Nueva York: Springer-Verlag. 